\title{
Integrated Production-Delivery Lot Sizing Model with Limited Production Capacity and Transportation Cost considering Overtime Work and Maintenance Time
}

\author{
Renqian Zhang $\mathbb{D}$ and Xuefang Sun \\ School of Economics and Management, Beihang University, Beijing 100191, China \\ Correspondence should be addressed to Renqian Zhang; zhangrenqian@buaa.edu.cn
}

Received 13 December 2017; Accepted 7 March 2018; Published 15 April 2018

Academic Editor: Eric Feulvarch

Copyright (C) 2018 Renqian Zhang and Xuefang Sun. This is an open access article distributed under the Creative Commons Attribution License, which permits unrestricted use, distribution, and reproduction in any medium, provided the original work is properly cited.

\begin{abstract}
An extension of the integrated production-delivery lot sizing model with limited production capacity and transportation cost is investigated. We introduce the factor of overtime work into the model to improve the manufacturer's production. In addition, when finishing a lot, the manufacturer has maintenance time to maintain and repair equipment for ensuring that the supply chain is operating continuously. By analyzing the integrated model, the solution procedure is provided to determine the optimal delivery and order policy. We conduct a numerical experiment and give sensitive analysis by varying some parameters to illustrate the problem and its solution procedure.
\end{abstract}

\section{Introduction}

One goal of supply chain management is to maximize profit or minimize cost. Traditionally, the retailer and manufacturer trade with each other based on the economic order quantity (EOQ) or economic production quantity (EPQ) model. Along with the diversification of marketing pattern, parties among the supply chain have realized the limitations and shortages of the EOQ and EPQ models. To satisfy current market demand, joint economic lot sizing (JELS) inventory model has been proposed to coordinate the productiondelivery strategy among suppliers, manufacturers, retailers, and so on. The aim of the model is to seek optimal production lot sizing based on integrated cost/profit function, rather than each party's individual objectives.

Goyal [1] first analyzed a joint economic lot sizing (JELS) inventory model with the assumption of an infinite production rate and obtained the single-vendor and singlebuyer optimal ordering strategy. Banerjee [2] relaxed Goyal's [1] model by taking production capacity into consideration and gave the corresponding optimal lot-for-lot policy. After that, Goyal [3] developed a more general JELS model in which the supplier retained a part of inventory in stock and the buyer received orders at regular time intervals. Goyal and Gupta [4] and Thomas and Griffin [5] further conducted related research. $\mathrm{Lu}$ [6] found a one-vendor and multibuyer integrated production-delivery model to coordinate the replenishment of different items. Subsequently, Hill [7] introduced the geometric growth factor as a decision variable into the integrated production-delivery model. For this integrated model, Hill [8] gave the optimal production and shipment solution.

Most early research on the EOQ model assumes that setup cost is constant or a fixed part of ordering cost. However, the Japanese have successfully introduced the "just-in-time" philosophy that reduces setup cost and time as much as possible. Subsequently, related research has been published to investigate the effects of investment in reducing setup cost. Porteus [9] analyzed an investment cost with the setup level and examined how the capital investment impacts on reducing setup cost in the standard EOQ model. Later, Porteus $[10,11]$ extended the research to the discounted EOQ model. Billington [12] presented an economic production quantity (EPQ) model where setup cost varied linearly or exponentially as a function of investment. Then, Kim et al. 
[13] extended the model of Billington [12] and Porteus [9] by analyzing the corresponding behavior under different setup cost reduction functions. Spence and Porteus [14] considered the impact of setup reduction on production capacity. Nasri et al. [15] considered decreasing setup cost as a decision variable in the EOQ model. Then, the extension of the setup reduction model was introduced into the economic manufacturing quantity (EMQ) model by Sarker and Coates [16]. Later, under the conditions of setup cost reduction, Huang [17] and Lou and Wang [18] determined the optimal policies of the integrated inventory model when payment is permitted delay. In recent years, Shahpouri et al. [19] developed an integrated vendor-buyer inventory model incorporating lead time and ordering cost reduction with a service-level constraint. In their model, it is suggested that decreasing lead time and reducing ordering cost can decrease the expected total cost. An integrated procurement production inventory model for defective products was studied by Priyan et al. [20] to simultaneously control setup and ordering cost under the JIT policy. Sarkar et al. [21] invented a two-echelon supply chain model with deterioration by considering the effects of setup cost reduction and quantity improvement. Our paper will follow the assumption that the setup cost is a function of investment.

So far, all the models mentioned above assumed that the transportation cost is part of the ordering cost. In reality, however, this assumption is unreasonable since transportation cost is impacted by many factors like the routing decisions, the selected shipment size, and others. There is a substantial amount of studies addressing the related problems. Baumol and Vinod [22] provided a simplified model that describes the essentials of freight shipment. They sought the minimization of inventory cost and maximization of profit, respectively, in their model. Then, Burns et al. [23] developed an analytic method to minimize the sum of transportation and inventory costs when items are distributed from a supplier to multiple customers. They compared the optimal decisions under two distribution strategies: direct shipping and peddling. New vendor-managed inventory systems that coordinated inventory and transportation decision in a stochastic setting were proposed by Çetinkaya and Lee [24]. Çetinkaya and Lee [25] utilized the methodology of Çetinkaya and Lee [24] to optimize the integrated inventory and outbound transportation model. In their model, the outbound distribution warehouse is operated by a third-party provider and freight quantities are consolidated. In addition, Toptal et al. [26] suggested the vendor-buyer coordinating model including inbound and outbound cargo capacity and coordinating transportation cost. Lee [27] incorporated the discounted freight rate into the EOQ model. Burwell et al. [28] constructed a price-dependent demand model with freight discounts. Swenseth and Godfrey [29] incorporated two freight rate functions into the inventory replenishment model to explore the impact on purchasing decisions. Subsequently, Ertogral et al. [30] developed optimal solution procedures for solving the integrated inventory model. They concluded that the explicit incorporation of transportation cost in the integrated inventory model has great effect on the production decisions. Then, Shu et al. [31] extended the production-delivery lot sizing model under the jointly exponentially distributed delivery time. Other related articles can be found in Kang and Kim [32], Hwang [33, 34], and Lee and Fu [35]. Recently, Makkar and Jha [36] developed a procurement-distribution model with freight discount for perishable product. A three-party supply chain system with quantity and freight discounts was provided by Ke et al. [37]. Jauhari and Winingsih [38] illustrated the supply chain coordination in a stochastic environment using freight discount by proposing four schedules. Birbil et al. [39] identified the set of purchase-transportation cost functions into an economic order quantity cost model. They proposed a Lipschitz optimization procedure for the problem.

In real production management, some manufacturers may have an insufficient production capacity due to being understaffed, possessing outdated equipment, and other restricted conditions. To meet the large demand, overtime work is the most common and relative effective method that many manufacturers usually adopt. Foxconn Technology Group is a classic case where the manager requires the staff to work several hours of overtime per day to improve the production rate. Another representative example is Toyota Motor Corporation. In August, Toyota announced that they would adopt "discretionary labor system" in December. This new measure can promote the enterprise's production capacity by encouraging workers to work overtime. In this paper, we attempt to extend the model of Shu et al. [31] by assuming that the manufacturer's production rate is smaller than the market demand rate. We take the factor of overtime work into consideration to increase the production rate per day.

The objective is to minimize the integrated average total cost of the two-stage supply chain, based on which the optimal delivery quantity, shipment number, and investment expenditure are obtained. It is obvious that decreasing the manufacturer's holding inventory can effectively reduce the integrated inventory cost. Hence, the best policy to minimize the integrated annual cost is that the manufacturer should have no excess inventory during each delivery interval; that is, the manufacturer's production quantity during each delivery interval equals the size of each shipment. Furthermore, to ensure the normal operation of this supply chain, the manufacturer does not immediately produce the next lot when each lot is finished but shuts down for a time interval to maintain and repair equipment. This consideration is very different from the previously mentioned literature. We developed an algorithm to solve the model in this paper which can yield globally optimal solution. Numerical examples and analysis are shown to prove the effectiveness of the algorithm. In addition, this paper follows previous research by assuming that the transportation cost is a ceiling function of each delivery quantity.

The remainder of this paper is organized as follows. In Section 2, we provide notations and assumptions. In Section 3, we present our model with the manufacturer's overtime work and transportation cost. In Section 4, we analyze the model and propose a solution procedure to minimize the integrated annual total cost. Numerical experiments and sensitive analysis are given in Section 5. Finally, the conclusion and the future extensions of the proposed model have been made in last section. 


\section{Notations and Assumptions}

This paper develops a long-term production-delivery model, where the manufacturer's production rate $R$ is smaller than demand rate $D$. To improve insufficient production capacity, the manufacturer proposes a rational overtime work strategy to increase production rate from $R$ to $(1+\alpha) R$, where $\alpha$ is the increasing production rate by working overtime. Unit production cost of the manufacturer is increased from $c$ to $c_{1}$ when workers work overtime. The retailer orders $Q$ units from the manufacturer per production run. However, if all $Q$ units goods are delivered at a time, not only will the manufacturer increase production pressure, but also the retailer will also increase holding cost. To avoid these dilemmas, each lot is divided into $n$ times for delivery, and the manufacturer's delivery pressure decreases to $q$ during each delivery interval. Thus, the produced total quantity per lot is $Q=n q$ and the delivery cycle is $T=q / D$.

The model runs as follows. When the retailer orders $Q$ units goods from the manufacturer, the manufacturer produces $q$ units by overtime and then delivers the first $q$ units goods to the retailer. The first delivery point is $q /(1+$ $\alpha) R$ (point F). The manufacturer synchronizes working $t$ by overtime work and then working $T-t$ normally and continuously during cycles $[q /(1+\alpha) R, q /(1+\alpha) R+T],[q /(1+$ $\alpha) R+T, q /(1+\alpha) R+2 T], \ldots$, and $[q /(1+\alpha) R+(n-2) T, q /(1+$ $\alpha) R+(n-1) T]$. At point $q /(1+\alpha) R+(n-1) T$ (point A), the manufacturer finishes a lot $n q$ and shuts down for a time interval $\Delta t$ to maintain and repair equipment. The following notations and assumptions are used to develop the integrated inventory model.

\section{Notations}

$q_{0}$ : the capacity per vehicle

$E$ : transportation cost per vehicle

$K$ : expenditure per day to operate the planning ordering system between the manufacturer and the retailer in each ordering cycle

$U(K)$ : the retailer's setup cost per shipment

$A_{m}$ : the manufacturer's setup cost for each production run

$A_{s}$ : the manufacturer's opportunity cost per production run, including maintenance cost, the lost profit, and goodwill loss caused by shutdown

$h_{m}$ : inventory holding cost per unit per time for the manufacturer

$h_{r}$ : inventory holding cost per unit per time for the retailer

$C_{m}$ : the manufacturer's average total cost

$C_{r}$ : the retailer's average total cost

$J$ : the integrated inventory model's average total cost

Assumptions. (1) According to the description of Billington [12], the capital investment impacts on the reduction of setup cost to minimize the total cost of the EOQ model.
In this paper, to obtain the optimal cost of the model, we follow Billington's [12] assumption that the retailer's setup cost per shipment $U(K)$ is a negative exponential function of operating expenditure $K$ :

$$
U(K)=U_{0} e^{-\lambda K},
$$

where $U_{0}$ is original order setup cost per shipment and $\lambda$ is a constant parameter.

(2) Demand rate $D$ is constant over time, and the manufacturer's production rate $R$ is smaller than the demand rate $D$. The manufacturer can increase the production rate to $(1+\alpha) R$ by working overtime. To make the problem tractable, we assume that the production rate by working overtime is larger than the demand rate; that is, $(1+\alpha) R>D$.

(3) With the increase of operating time in each ordering cycle, the equipment wear is more serious. The equipment wear can to some extent reduce the production capacity. To make sure that the equipment's production rate is unchangeable and equipment is running normally, the manufacturer reserves maintenance time to maintain and repair equipment when each lot is finished. To make this near to the actual situation, we assume that maintenance time is a nondecreasing function of ordering cycle

$$
\Delta t(n T)=\gamma \log (n T+C)+\eta(n T)^{2},
$$

where $\gamma, \eta$, and $C$ are constants and $\lambda, \eta, C>0$. The equipment still needs to be maintained, even if nothing is produced; that is, $\Delta t(\Delta t)>0$ when the production time $n T-\Delta t$ is zero. For maintenance time $\Delta t$, we can find a linear function $\Delta t^{\prime}=\beta n T+\Delta t(\Delta t)$, where $\beta$ is a constant and $0<\beta<1$, subject to $\Delta t \geq \Delta t^{\prime}$ for all $n T$. By analyzing $\Delta t \geq \Delta t^{\prime}$, we obtain $\Delta t / n T>\beta$.

(4) Shortages are not allowed.

(5) To ensure the continuous production operation, the manufacturer should produce $q /(1+\alpha) R$ units of goods in advance.

\section{Mathematical Model}

Figure 1 illustrates the inventory profiles for both the manufacturer and the retailer over one replenishment cycle. To decrease the manufacturer's holding cost, the manufacturer adopts a reasonable overtime strategy to make the manufacturer's production quantity during each delivery interval equal to the size of each shipment; that is, $(1+\alpha) R t+R(T-t)=$ $D T$. Therefore, the length of overtime during each delivery interval is $t=(D-R) T / \alpha R=(D-R) q / \alpha R D$.

The average integrated total cost consists of the manufacturer's and the retailer's respective average total costs. Next, we separately discuss them.

3.1. The Manufacturer's Average Total Cost. The manufacturer's average total cost consists of holding cost, setup cost, and production cost. The total number of shipments in each ordering cycle and the delivery quantity at each delivery point are two decision variables for the manufacturer. Hence, the manufacturer's average total cost is a function of $n$ and $q$. In Figure 1, the polygonal line OEDB illustrates the accumulated 

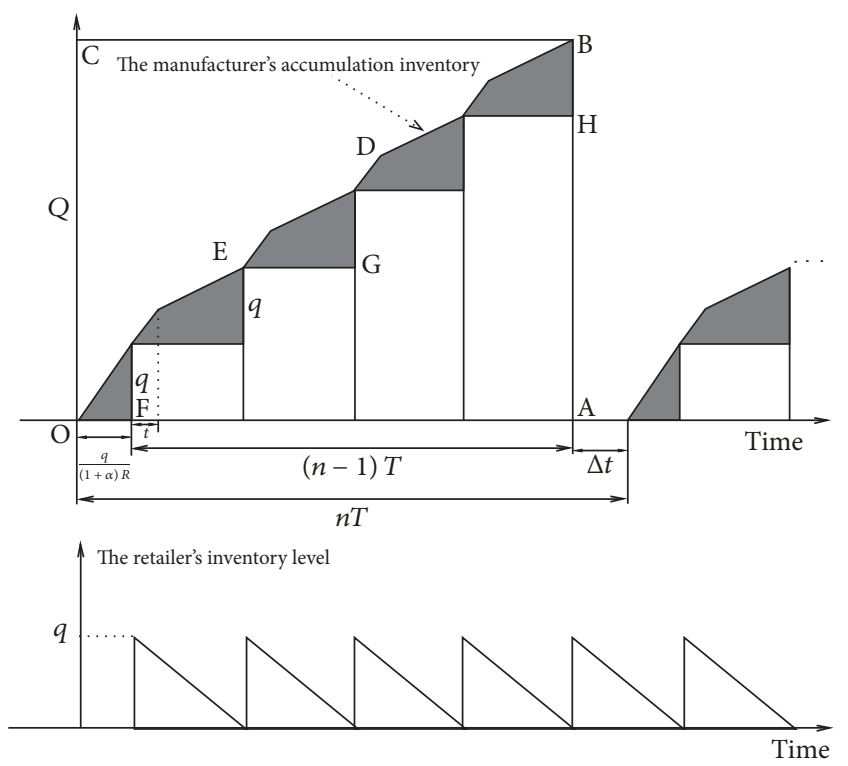

FIGURE 1: The manufacturer and retailer's on-hand inventory.

inventory level of the manufacturer. The trapezoid region AFGH depicts the total depletion of the manufacturer's inventory. The polygonal line OEDB intersects the trapezoid region $\mathrm{AFGH}$ at each delivery point. In other words, the manufacturer has no excess inventory at each delivery point; that is, the manufacturer's production quantity exactly equals the manufacturer's delivery volume during each delivery interval. In Figure 1, we illustrate that the shaded region OEDBHGF represents the manufacturer's total accumulated inventory in each ordering cycle.

The shaded region OEDBHGF

$=$ the rectangle area $\mathrm{OABC}$

- the polygon area OCBDE

- the stair-shaped area AHGF $=\left[\frac{D T}{(1+\alpha) R}\right.$

$+(n-1) T] n D T-\left[\frac{2 D T}{(1+\alpha) R}(n-1)+(n-1) t\right.$

$+(n-1)(n-2) T] \frac{(1+\alpha) R t}{2}-\left[\frac{2 D T}{(1+\alpha) R}(n-1)\right.$

$\left.+(n-1) t+(n-1)^{2} T\right] \frac{R(T-t)}{2}-\frac{q^{2}}{2(1+\alpha) R}$

$-\frac{D T^{2}(n-1) n}{2}=\left[\frac{D T}{(1+\alpha) R}+(n-1) T\right] n D T$

$-\frac{n(n-1) q T}{2}-\frac{q^{2}}{2(1+\alpha) R}$

$-\frac{R(\alpha t+T)}{2}\left[\frac{2 q(n-1)}{(1+\alpha) R}+(n-1)(n-2) T\right.$

$+(n-1) t]-\frac{R(T-t)(n-1) T}{2}$.
As a result, the manufacturer's average holding cost

$$
\begin{aligned}
= & \frac{h_{m}}{n T}\left\{\left[\frac{D T}{(1+\alpha) R}+(n-1) T\right] n D T-\frac{n(n-1) q T}{2}\right. \\
& -\frac{q^{2}}{2(1+\alpha) R}-\frac{R(\alpha t+T)}{2}\left[\frac{2 q(n-1)}{(1+\alpha) R}\right. \\
& +(n-1)(n-2) T+(n-1) t] \\
& \left.-\frac{R(T-t)(n-1) t}{2}\right\}=h_{m} q\left[\frac{D}{(1+\alpha) R n}\right. \\
& \left.-\frac{(n-1) D}{2 n \alpha R}+\frac{(1+\alpha)(n-1)}{n \alpha}-\frac{(1+\alpha)(n-1) R}{2 n \alpha D}\right] .
\end{aligned}
$$

The manufacturer's average setup cost is $A_{m} / n T=A_{m} D / n q$.

The manufacturer's average opportunity cost is $A_{s} / n T=$ $A_{s} D / n q$.

According to the discussion above and Figure 1, the total production cost in each ordering cycle is $c_{1}(1+\alpha) R[q /(1+$ $\alpha) R+(n-1) t]+c R(n-1)(T-t)$. Thus, the manufacturer's average production cost is $c_{1} D / n+\left[c_{1}(1+\alpha)-c\right](n-1)(D-$ $R) / n \alpha+c R(n-1) / n$.

Combined with the discussion above, we obtain the manufacturer's average cost

$$
\begin{aligned}
& C_{m}(q, n)=h_{m} q\left[\frac{D}{2(1+\alpha) R n}-\frac{(n-1) D}{2 n \alpha R}\right. \\
& \left.+\frac{(1+\alpha)(n-1)}{n \alpha}-\frac{(1+\alpha)(n-1) R}{2 n \alpha D}\right]+\frac{A_{m} D}{n q} \\
& +\frac{A_{s} D}{n q}+\frac{c_{1} D}{n}+\frac{\left[c_{1}(1+\alpha)-c\right](n-1)(D-R)}{n \alpha} \\
& +\frac{c(n-1) R}{n} .
\end{aligned}
$$

For convenience, let $F=D / 2(1+\alpha) R n-(n-1) D / 2 n \alpha R+$ $(1+\alpha)(n-1) / n \alpha-(1+\alpha)(n-1) R / 2 n \alpha D$. The manufacturer's average total cost becomes

$$
\begin{aligned}
C_{m}(q, n)= & h_{m} q F+\frac{\left(A_{m}+A_{s}\right) D}{n q}+\frac{c_{1} D}{n} \\
& +\frac{\left[c_{1}(1+\alpha)-c\right](n-1)(D-R)}{n \alpha} \\
& +\frac{c(n-1) R}{n} .
\end{aligned}
$$

Next, we discuss the retailer's average total cost.

3.2. The Retailer's Average Total Cost. In most inventory models, transportation cost is fixed or a continuous function of ordering quantity. In this paper, we view transportation cost as a ceiling function with respect to the number of vehicles used to transport goods. According to notations in Section 2 , we know that the capacity per vehicle is $q_{0}$. Hence, 
when the manufacturer delivers $q$ units of goods to the retailer at delivery point, the retailer needs to pay $\left\lceil\left(q / q_{0}\right) E\right\rceil$ for transportation.

The retailer's average inventory cost consists of transportation cost, setup cost, holding cost, and expenditure cost. Hence, the retailer has two decision variables: delivery quantity at each delivery point and operating expenditure in each delivery cycle. Obviously, the retailer's average total cost is a function of $q$ and $K$. According to the discussion above, the retailer's average total cost can be represented as

$$
\begin{aligned}
C_{r}(q, K)= & \frac{1}{T}\left[\left\lceil\frac{q}{q_{0}}\right\rceil E+U_{0} e^{-\lambda K}+h_{r} \int_{0}^{T} q-D t d t\right] \\
& +K=\left\lceil\frac{q}{q_{0}}\right\rceil \frac{E D}{q}+\frac{D U_{0} e^{-\lambda K}}{q}+\frac{h_{r} q}{2}+K .
\end{aligned}
$$

Now, we elaborate on the integrated average cost for both the manufacturer and the retailer.

3.3. The Integrated Average Total Cost. The manufacturer and the retailer work together in a cooperative manner to minimize the integrated average total cost. Thus, the integrated average total cost consists of the manufacturer's average total cost and the retailer's average total cost. According to Sections 3.1 and 3.2, we know that the integrated average total cost has three decision variables, including the total number of shipments in each ordering cycle, the delivery quantity at each delivery point, and the operating expenditure in each ordering cycle. Hence, the integrated average total cost is a function of $q, n$, and $K$. The integrated average total cost can be written as

$$
\begin{aligned}
J(q, n, K)= & h_{m} q F+\frac{\left(A_{m}+A_{s}\right) D}{n q}+\frac{c_{1} D}{n} \\
& +\frac{\left[c_{1}(1+\alpha)-c\right](n-1)(D-R)}{n \alpha} \\
& +\frac{c(n-1) R}{n}+\left\lceil\frac{q}{q_{0}}\right\rceil \frac{E D}{q}+\frac{D U_{0} e^{-\lambda K}}{q} \\
& +\frac{h_{r} q}{2}+K .
\end{aligned}
$$

\section{Analysis and Optimal Solution}

The objective of this model is to minimize the integrated average total cost $J(q, n, K)$ defined by (8). To find the optimal solution, we analyze the first- and second-order partial derivatives of $J(q, n, K)$ with respect to $K$.

$$
\begin{gathered}
\frac{\partial J(q, n, K)}{\partial K}=-\frac{\lambda D U_{0} e^{-\lambda K}}{q}+1 . \\
\frac{\partial^{2} J(q, n, K)}{\partial^{2} K^{2}}=\frac{\lambda^{2} D U_{0} e^{-\lambda K}}{q} .
\end{gathered}
$$

Obviously, $\lambda^{2} D U_{0} e^{-\lambda K} / q>0$ holds for all $K$, which proves that function $J(q, n, K)$ is convex in $K$. Setting (9) to be zero, we obtain

$$
K=\frac{1}{\lambda} \ln \frac{\lambda D U_{0}}{q} .
$$

By inspecting Section 2, we know that $n$ represents the total number of shipments in each ordering cycle. Hence, $n$ is a positive integer. We derive the range of $n$ as follows.

Proposition 1. The total number of shipments in each ordering cycle is an integer between 1 and $\lfloor 1 / \beta-D / \beta(1+\alpha) R\rfloor$.

\section{Proof. See Appendix A.}

For the convenience of the discussion below, let $\bar{n}=$ $\lfloor 1 / \beta-D / \beta(1+\alpha) R\rfloor$. There is an implicit assumption to ensure the feasibility of the model: $\bar{n} \geq 1$.

Now, we analyze the first-order partial derivative of $J(q, n, K)$ with respect to $q$ for a certain $n$.

Because of the discontinuity of $\left\lceil q / q_{0}\right\rceil$, the function $J(q, n, K)$ with respect to $q$ is discontinuous. It is difficult to obtain the optimal delivery quantity by calculating the firstand second-order partial derivative of $J(q, n, K)$ with respect to $q$. We introduce a parameter $\varepsilon$, subject to $q / q_{0}+\varepsilon$ being an integer, where $0<\varepsilon<1$. Hence, the integrated average total cost becomes

$$
\begin{aligned}
J_{\varepsilon}(q, n, K)= & h_{m} q F+\frac{\left(A_{m}+A_{s}\right) D}{n q}+\frac{c_{1} D}{n} \\
& +\frac{\left[c_{1}(1+\alpha)-c\right](n-1)(D-R)}{n \alpha} \\
& +\frac{c(n-1) R}{n}+\left(\frac{q}{q_{0}}+\varepsilon\right) \frac{E D}{q} \\
& +\frac{D U_{0} e^{-\lambda K}}{q}+\frac{h_{r} q}{2}+K \\
= & h_{m} q F+\frac{\left(A_{m}+A_{s}\right) D}{n q}+\frac{c_{1} D}{n} \\
& +\frac{\left[c_{1}(1+\alpha)-c\right](n-1)(D-R)}{n \alpha} \\
& +\frac{c(n-1) R}{n}+\frac{E D}{q_{0}}+\varepsilon \frac{E D}{q} \\
& +\frac{D U_{0} e^{-\lambda K}}{q}+\frac{h_{r} q}{2}+K .
\end{aligned}
$$

Obviously, $J_{\varepsilon}(q, n, K)$ is continuous with respect to $q$, and we can obtain the first- and second-order partial derivatives of $J_{\varepsilon}(q, n, K)$ with respect to $q$, respectively, as

$$
\begin{aligned}
\frac{\partial J_{\varepsilon}(q, n, K)}{\partial q}= & -\frac{\left(A_{m}+A_{s}\right) D}{n q^{2}}+h_{m} F-\frac{\varepsilon E D}{q^{2}} \\
& -\frac{D U_{0} e^{-\lambda K}}{q^{2}}+\frac{h_{r}}{2},
\end{aligned}
$$




$$
\begin{aligned}
\frac{\partial J_{\varepsilon}^{2}(q, n, K)}{\partial q^{2}}= & \frac{2\left(A_{m}+A_{s}\right) D}{n q^{3}}+\frac{2 \varepsilon E D}{q^{3}} \\
& +\frac{2 D U_{0} e^{-\lambda K}}{q^{3}} .
\end{aligned}
$$

From (13), we know that $2\left(A_{m}+A_{s}\right) D / n q^{3}+2 \varepsilon E D / q^{3}+$ $2 D U_{0} e^{-\lambda K} / q^{3}>0$ for all $q>0$, which demonstrates that $J_{\varepsilon}(q, n, K)$ is convex for $q>0$. By setting (12) to be zero and combining with (10), we derive

$$
\begin{aligned}
& q^{(\varepsilon)} \\
& =\frac{2\left[\left(A_{m}+A_{s}\right) D / n+\varepsilon E D\right]}{-1 / \lambda+\sqrt{1 / \lambda^{2}+4\left[\left(A_{m}+A_{s}\right) D / n+\varepsilon E D\right]\left(h_{m} F+h_{r} / 2\right)}} .
\end{aligned}
$$

Proposition 2 determines the optimal value of $\varepsilon$ for minimizing the integrated average total cost $J_{\varepsilon}$.

Proposition 2. The integrated average total cost can be at its minimum when $\varepsilon=0$; that is, when the size of each shipment is integer multiple of the capacity per vehicle, the integrated average total cost can reach to the minimum for a certain $n$.

Proof. See Appendix B.

Note

$$
q=k q_{0}
$$

where $k$ is a positive integer variable. We rewrite the integrated average total cost $J(q, n, K)$ as

$$
\begin{aligned}
J(k, n, K)= & h_{m} k q_{0} F+\frac{\left(A_{m}+A_{s}\right) D}{n k q_{0}}+\frac{c_{1} D}{n} \\
& +\frac{\left[c_{1}(1+\alpha)-c\right](n-1)(D-R)}{n \alpha} \\
& +\frac{c(n-1) R}{n}+\frac{E D}{q_{0}}+\frac{D U_{0} e^{-\lambda K}}{k q_{0}} \\
& +\frac{h_{r} k q_{0}}{2}+K .
\end{aligned}
$$

Combining (16) with (9), the first-order partial derivative of $J(q, n, K)$ with respect to $K$ becomes

$$
\frac{\partial J(k, n, K)}{\partial K}=-\frac{\lambda D U_{0} e^{-\lambda K}}{k q_{0}}+1 .
$$

Setting (17) to be zero. We derive

$$
K=\frac{1}{\lambda} \ln \frac{\lambda D U_{0}}{k q_{0}} .
$$

Next, incorporating with (18), we analyze the first-order partial derivative of $J(q, n, K)$ with respect to $k$ and set the result to be zero.

$$
\begin{aligned}
\frac{\partial J(k, n, K)}{\partial k}= & -\frac{\left(A_{m}+A_{s}\right) D}{n q_{0} k^{2}}+h_{m} F q_{0}-\frac{D U_{0} e^{-\lambda K}}{k^{2} q_{0}} \\
& +\frac{h_{r}}{2} \\
= & -\frac{\left(A_{m}+A_{s}\right) D}{n q_{0} k^{2}}+h_{m} F q_{0}-\frac{1}{k \lambda}+\frac{h_{r}}{2} \\
= & 0 .
\end{aligned}
$$

The solution is

$$
\begin{aligned}
& k \\
& n q_{0}\left[-1 / \lambda+\sqrt{1 / \lambda^{2}+\left(4\left(A_{m}+A_{s}\right) D / n\right)\left(h_{m} F+h_{r} / 2\right)}\right]
\end{aligned}
$$

For a given $n$, the local optimal solution of $k$ is given by (20) when it is an integer. Otherwise, let $k_{1}=\lfloor k\rfloor$ and $k_{2}=\lceil k\rceil$. Accordingly, we calculate the corresponding delivery quantity $q_{1}$, investment expenditure $K_{1}$, and integrated average total cost $J_{1}$ under $k_{1}$ and calculate $q_{2}, K_{2}$, and $J_{2}$ under $k_{2}$, combining with (18) and (15). As analyzing the above, we obtain the local optimal solution of $k$.

$$
\overline{k^{n}}= \begin{cases}k, & \text { if } k \text { is integer, } \\ k_{2}, & \text { if } k \text { is not integer and } J_{1}>J_{2}, \\ k_{1}, & \text { if } k \text { is not integer and } J_{1} \leq J_{2} .\end{cases}
$$

Combined with (18), (20), (21), and (15), for a certain $n$, the local optimal solutions $q$ and $K$ are solved. To prevent any potential confusion, let $\overline{q^{n}}$ and $\overline{K^{n}}$ represent the local optimal solutions $q$ and $K$, respectively. Substituting $\overline{q^{n}}$ and $\overline{K^{n}}$ into (8), we get the local minimum average total cost and note it as $\overline{J^{n}}$.

As a summary, the procedure for determining the optimal inventory policy can be described as follows.

Step 1. Determine $\bar{n}$ from Proposition 1.

Step 2. Let $n=1$ and calculate the local optimal solutions $\overline{k^{1}}, \overline{q^{1}}, \overline{K^{1}}$, and $\overline{J^{1}}$, respectively, combined with (20), (21), (15), (18), and (8).

Step 3. Let $n=n+1$. If $n \leq \bar{n}$, determine the local optimal solutions $\overline{k^{n}}, \overline{q^{n}}, \overline{K^{n}}$, and $\overline{J^{n}}$, respectively, combined with (20), (21), (15), (18), and (8). Then, proceed to the same calculation procedure in this step. Otherwise, proceed to the next step.

Step 4. Compare all the local optimal costs: $\overline{J^{1}}, \overline{J^{2}}, \ldots, \overline{J^{n}}$. The lowest one of them is the optimal average total cost for our problem and is denoted as $J^{*}$. The corresponding delivery quantity, the total number of shipments, and the operating expenditure are noted as $q^{*}, n^{*}$, and $K^{*}$, respectively. 
TABLE 1: The optimal decision of the integrated model by varying $\alpha$.

\begin{tabular}{lcccccc}
\hline$\alpha$ & $n^{*}$ & $k^{*}$ & $q^{*}$ & $K^{*}$ & $J^{*}$ & $C_{m}{ }^{*}$ \\
\hline 0.35 & 1 & 3 & 90 & 24.0795 & 2181.3017 & 1588.8889 \\
0.4 & 2 & 2 & 60 & 28.1341 & 1976.2055 & 1454.7381 \\
0.6 & 4 & 2 & 60 & 28.1341 & 1858.7383 & 1337.2708 \\
0.7 & 5 & 2 & 60 & 28.1341 & 1831.8383 & 1310.3709 \\
0.8 & 6 & 1 & 30 & 35.0656 & 1736.6628 & 521.4674 \\
\hline
\end{tabular}

TABLE 2: The optimal decision of the integrated model by varying $\beta$.

\begin{tabular}{lcccccrr}
\hline$\beta$ & $n^{*}$ & $k^{*}$ & $q^{*}$ & $K^{*}$ & $J^{*}$ & $C_{m}{ }^{*}$ \\
\hline 0.01 & 10 & 1 & 30 & 35.0656 & 1729.4727 & 1276.0738 & $C_{r}{ }^{*}$ \\
0.03 & 3 & 2 & 30 & 28.1341 & 1914.2928 & 1392.8254 & 521.4674 \\
0.05 & 2 & 2 & 60 & 28.1341 & 1976.2055 & 1454.7381 \\
0.06 & 1 & 3 & 90 & 24.0795 & 2175.3493 & 1582.9365 & 521.4674 \\
0.1 & 1 & 3 & 90 & 24.0795 & 2175.3493 & 1582.9365 & 592.4128 \\
\hline
\end{tabular}

TABLE 3: The optimal decision of the integrated model by varying $\lambda$.

\begin{tabular}{|c|c|c|c|c|c|c|c|}
\hline$\lambda$ & $n^{*}$ & $k^{*}$ & $q^{*}$ & $K^{*}$ & $J^{*}$ & $C_{m}{ }^{*}$ & $C_{r}{ }^{*}$ \\
\hline 0.01 & 2 & 2 & 60 & 51.0826 & 2089.1540 & 1454.7381 & 634.4159 \\
\hline 0.05 & 2 & 2 & 60 & 42.4053 & 2000.4767 & 1454.7381 & 545.7386 \\
\hline 0.1 & 2 & 2 & 60 & 28.1341 & 1976.2055 & 1454.7381 & 521.4674 \\
\hline 0.2 & 2 & 2 & 60 & 17.5328 & 1960.6042 & 1454.7381 & 505.8661 \\
\hline 0.4 & 2 & 2 & 60 & 10.4993 & 1951.0707 & 1454.7381 & 496.3326 \\
\hline 0.8 & 2 & 2 & 60 & 6.1161 & 1945.4375 & 1454.7381 & 490.6994 \\
\hline
\end{tabular}

\section{Numerical Examples and Sensitivity Analysis}

Consider an example where the following parameters are known: $D=100$ units/day, $R=80$ units/day, $h_{m}=$ $\$ 4 /$ unit/day, $h_{r}=\$ 5 /$ unit/day, $E=\$ 100 /$ vehicle, $U_{0}=\$ 100 /$ shipment, $c=\$ 10 /$ unit, $c_{1}=\$ 12 /$ unit, $A_{m}=\$ 100 /$ order, $A_{s}=\$ 100 /$ order, $q_{0}=30$ units/vehicle, $\lambda=0.1, \alpha=0.4$, and $\beta=0.05$.

We illustrate the solution procedure as follows:

(1) $\bar{n}=2$ is obtained from Step 1.

(2) $n=1, \overline{k^{1}}=3, \overline{q^{1}}=90, \overline{K^{1}}=24.0795, \overline{J^{1}}=2112.3493$.

(3) $n=2, \overline{k^{2}}=2, \overline{q^{2}}=60, \overline{K^{2}}=28.1341, \overline{J^{2}}=1976.2055$.

(4) Comparing $\overline{J^{1}}, \overline{J^{2}}$, we know $\overline{J^{2}}$ is the minimum. Then, the optimal delivery size is $q^{*}=60$, the optimal expenditure is $K^{*}=28.1341$, the optimal number of deliveries is $n^{*}=2$, and the lowest cost is $J^{*}=1976.2055$.

In the model, there are many parameters involved, including the indemnify rate $\alpha$, the parameter of maintenance time $\beta$, and the parameter of setup cost for the retailer $\lambda$. To better understand the effect of this model on the manufacturer and the retailer's decision, sensitivity analysis is given by varying some parameters while fixing other parameters.

Table 1 presents the impact of indemnify rate variability on the integrated inventory model developed in this paper. The optimal number of shipments is increasing when the indemnify rate of the manufacturer $\alpha$ increases. Conversely, the optimal delivery quantity $q^{*}$ and the optimal integrated average total cost $J^{*}$ are decreasing as the indemnify rate $\alpha$ increases. From Table 1 , it can be observed that the integrated average cost decreases from 2181.3017 to 1976.2055 when the increased rate of production rate by working overtime increases from 0.35 to 0.4 . However, the integrated average cost decreases from 1858.7383 to 1831.8383 when the increasing rate increases from 0.6 to 0.7 . It shows that the influence of overtime on the model declines as $\alpha$ increases. Thus, the manufacturer should adopt appropriate overtime work policy in the production operation.

Table 2 analyzes how the parameter of maintenance time $\beta$ impacts the optimal production-delivery strategy of the integrated model. Clearly, the manufacturer's and the retailer's costs increase as the maintenance time is extended. Meanwhile, the integrated average cost increases, too. When the manufacturer spends more time maintaining and repairing equipment in each ordering cycle, the number of deliveries decreases to minimize the integrated average cost. Table 2 also shows that more capital is lost when the percentage of maintenance time increases. Hence, in order to prevent extensive equipment damage, the proper use of equipment is necessary in the production process.

In Table 3, we obtain the reverse phenomenon compared with Table 2. According to assumption (1), the retailer's setup 
cost per shipment $U(K)$ is a negative exponential function of operating expenditure $K$. Since a large $\lambda$ brings large fluctuation of setup cost for fixed $K$, the integrated average cost decreases sharply as $\lambda$ increases. Simultaneously, the optimal operating expenditure $K^{*}$ and the retailer's cost to a great extent decrease when $\lambda$ increases. However, the reduction of setup cost has no influence on the optimal number of shipments $n^{*}$.

\section{Conclusion}

In this paper, we formulate an integrated production-delivery lot sizing model with a manufacturer and a retailer. Considering capacity constraints, the manufacturer adopts a proper overtime strategy to improve his production rate and to decrease holding cost. To ensure the supply chain operating normally and continuously, a time interval is given to maintain and repair equipment when each lot is finished. Besides, considering that both the manufacturer and the retailer bear large cost pressures when a batch goods are delivered at a time, each lot is divided into $n$ times for delivery. Based on Huang [17] and Lou and Wang [18], the article assumes that the retailer's setup cost is a negative exponential function of operating expenditure. In addition, transportation cost in this model is assumed to be a ceiling function of delivery quantity. Then, we analyze the integrated average total cost and give a solution procedure to obtain the optimal production-delivery strategy. Finally, numerical examples and sensitivity analysis are presented to illustrate the model and its solution procedure.

However, this model has several limitations. For instance, in real business activities, most production-delivery systems have more than one retailer and the demand rate is usually uncertain/stochastic. These problems should be of interest for future research. Beyond that, in real cases, when the manufacturer suffers from the capacity constraints problem, they might adopt overtime and emergency replenishment to satisfy the demand rate. This also should be considered in future research.

\section{Appendix}

\section{A. Proof of Proposition 1}

According to assumption (3), we deduce the following:

$$
\begin{aligned}
& \frac{\Delta t}{n T}>\beta \Longrightarrow \\
& \frac{n T-(n-1) T-q /(1+\alpha) R}{n T}>\beta \Longrightarrow \\
& T-\frac{q}{(1+\alpha) R}>\beta n T \Longrightarrow \\
& n<\frac{1}{\beta}-\frac{D}{\beta(1+\alpha) R} .
\end{aligned}
$$

Since $n$ represents the total number of shipments in each ordering cycle, $n$ is a positive integer and is at least 1 . Hence, $n$ is an integer between 1 and $\lfloor 1 / \beta-D / \beta(1+\alpha) R\rfloor$.

\section{B. Proof of Proposition 2}

First, substituting (14) and (10) into (11), we get

$$
\begin{aligned}
J_{\varepsilon}\left(q^{(\varepsilon)}, n, K\right) & h_{m} q^{(\varepsilon)} F+\frac{\left(A_{m}+A_{s}\right) D}{n q^{(\varepsilon)}}+\frac{c_{1} D}{n} \\
& +\frac{\left[c_{1}(1+\alpha)-c\right](n-1)(D-R)}{n \alpha}+\frac{c(n-1) R}{n} \\
& +\frac{E D}{q_{0}}+\frac{\varepsilon E D}{q^{(\varepsilon)}}+\frac{1}{\lambda}+\frac{h_{r} q^{(\varepsilon)}}{2}+K \\
= & \sqrt{\frac{1}{\lambda^{2}}+4\left[\frac{\left(A_{m}+A_{s}\right) D}{n}+\varepsilon E D\right]\left(h_{m} F+\frac{h_{r}}{2}\right)} \\
& +\frac{c_{1} D}{n}+\frac{\left[c_{1}(1+\alpha)-c\right](n-1)(D-R)}{n \alpha} \\
& +\frac{c(n-1) R}{n}+\frac{E D}{q_{0}}+\frac{1}{\lambda}+K .
\end{aligned}
$$

Clearly, this result is a function with respect to $\varepsilon$. The firstorder partial derivative of this function with respect to $\varepsilon$ is

$$
\begin{aligned}
& \frac{\partial J_{\varepsilon}}{\partial \varepsilon} \\
& =\frac{2 E D\left(h_{m} F+h_{r} / 2\right)}{\sqrt{1 / \lambda^{2}+4\left[\left(A_{m}+A_{s}\right) D / n+\varepsilon E D\right]\left(h_{m} F+h_{r} / 2\right)}} \\
& >0 .
\end{aligned}
$$

Hence, $J_{\varepsilon}$ is an increasing function of $\varepsilon$, where $0 \leq \varepsilon<1$. When $\varepsilon=0$, the integrated average total cost $J$ reaches to the minimum and $q$ is correctly integer multiple of $q_{0}$.

\section{Conflicts of Interest}

No potential conflicts of interest were reported by the authors.

\section{Acknowledgments}

This work was supported by the National Natural Science Foundation of China under Grant no. 71571006.

\section{References}

[1] S. K. Goyal, "An integrated inventory model for a singles upplier-single customer problem," International Journal of Production Research, vol. 15, no. 1, pp. 107-111, 1977.

[2] A. Banerjee, "A joint economic-lot-size model for purchaser and vendor," Decision Sciences, vol. 17, no. 3, pp. 292-311, 1986. 
[3] S. K. Goyal, "A joint economic-lot-size model for purchaser and vendor: a comment," Decision Sciences, vol. 19, no. 1, pp. 236241, 1988.

[4] S. K. Goyal and Y. P. Gupta, "Integrated inventory models: The buyer-vendor coordination," European Journal of Operational Research, vol. 41, no. 3, pp. 261-269, 1989.

[5] D. J. Thomas and P. M. Griffin, "Coordinated supply chain management," European Journal of Operational Research, vol. 94, no. 1, pp. 1-15, 1996.

[6] L. Lu, "A one-vendor multi-buyer integrated inventory model," European Journal of Operational Research, vol. 81, no. 2, pp. 312323, 1995.

[7] R. M. Hill, “The single-vendor single-buyer integrated productioninventory model with a generalised policy," European Journal of Operational Research, vol. 97, no. 3, pp. 493-499, 1997.

[8] R. M. Hill, "The optimal production and shipment policy for the singke-vendor singlebuyer integrated production-inventory problem," International Journal of Production Research, vol. 37, no. 11, pp. 2463-2475, 1999.

[9] E. L. Porteus, "Investing in reduced setups in the EOQ model," Management Science, vol. 31, no. 8, pp. 998-1010, 1985.

[10] E. L. Porteus, "Investing in new parameter values in the discounted EOQ model," Naval Research Logistics Quarterly, vol. 33, no. 1, pp. 39-48, 1986.

[11] E. L. Porteus, "Optimal lot sizing, process quality improvement and setup cost reduction," Operations Research, vol. 34, no. 1, pp. 137-144, 1986.

[12] P. J. Billington, "The classic economic production quantity model with setup cost as a function of capital expenditure," Decision Sciences, vol. 18, no. 1, pp. 25-42, 1987.

[13] S. L. Kim, J. C. Hayya, and J. Hong, "Setup Reduction in the Economic Production Quantity Model," Decision Sciences, vol. 23, no. 2, pp. 500-508, 1992.

[14] A. M. Spence and E. L. Porteus, "SETUP REDUCTION AND INCREASED EFFECTIVE CAPACITY.," Management Science, vol. 33, no. 10, pp. 1291-1301, 1987.

[15] F. Nasri, J. F. Affisco, and M. J. Paknejad, "Setup cost reduction in an inventory model with finite-range stochastic lead times," International Journal of Production Research, vol. 28, no. 1, pp. 199-212, 1990.

[16] B. R. Sarker and E. R. Coates, "Manufacturing setup cost reduction under variable lead times and finite opportunities for investment," International Journal of Production Economics, vol. 49, no. 3, pp. 237-247, 1997.

[17] C.-K. Huang, "An integrated inventory model under conditions of order processing cost reduction and permissible delay in payments," Applied Mathematical Modelling, vol. 34, no. 5, pp. 1352-1359, 2010.

[18] K.-R. Lou and W.-C. Wang, "A comprehensive extension of an integrated inventory model with ordering cost reduction and permissible delay in payments," Applied Mathematical Modelling, vol. 37, no. 7, pp. 4709-4716, 2013.

[19] S. Shahpouri, P. Fattahi, A. Arkan, and K. Parsa, "Integrated vendor-buyer cooperative inventory model with controllable lead time, ordering cost reduction, and service-level constraint," The International Journal of Advanced Manufacturing Technology, pp. 1-10, 2012.

[20] S. Priyan, M. Palanivel, and R. Uthayakumar, "Integrated procurement-production inventory model for defective items with variable setup and ordering cost," OPSEARCH, vol. 52, no. 4, pp. 692-713, 2015.
[21] B. Sarkar, A. Majumder, M. Sarkar, B. K. Dey, and G. Roy, "Two-echelon supply chain model with manufacturing quality improvement and setup cost reduction," Journal of Industrial and Management Optimization, vol. 13, no. 2, pp. 1085-1104, 2017.

[22] W. J. Baumol and H. D. Vinod, "An inventory theretic model of freight transport demand," Management Science, vol. 16, no. 7, pp. 413-421, 1970.

[23] L. D. Burns, R. W. Hall, D. E. Blumenfeld, and C. F. Daganzo, "Distribution strategies that minimize transportation and inventory costs," Operations Research, vol. 33, no. 3, pp. 469490, 1985.

[24] S. Çetinkaya and C.-Y. Lee, "Stock replenishment and shipment scheduling for vendor-managed inventory systems," Management Science, vol. 46, no. 2, pp. 217-232, 2000.

[25] S. 1. Çetinkaya and C.-Y. Lee, "Optimal outbound dispatch policies: modeling inventory and cargo capacity," Naval Research Logistics (NRL), vol. 49, no. 6, pp. 531-556, 2002.

[26] A. Toptal, S. Çetinkaya, and C.-Y. Lee, "The Buyer-Vendor coordination problem: Modeling inbound and outbound cargo capacity and costs," Institute of Industrial Engineers (IIE). IIE Transactions, vol. 35, no. 11, pp. 987-1002, 2003.

[27] C.-Y. Lee, "The economic order quantity for freight discount costs," Institute of Industrial Engineers (IIE). IIE Transactions, vol. 18, no. 3, pp. 318-320, 1986.

[28] T. H. Burwell, D. S. Dave, K. E. Fitzpatrick, and M. R. Roy, "Economic lot size model for price-dependent demand under quantity and freight discounts," International Journal of Production Economics, vol. 48, no. 2, pp. 141-155, 1997.

[29] S. R. Swenseth and M. R. Godfrey, "Incorporating transportation costs into inventory replenishment decisions," International Journal of Production Economics, vol. 77, no. 2, pp.113-130, 2002.

[30] K. Ertogral, M. Darwish, and M. Ben-Daya, "Production and shipment lot sizing in a vendor-buyer supply chain with transportation cost," European Journal of Operational Research, vol. 176, no. 3, pp. 1592-1606, 2007.

[31] M.-H. Shu, J.-C. Huang, and Y.-C. Fu, "A production-delivery lot sizing policy with stochastic delivery time and in consideration of transportation cost," Applied Mathematical Modelling, vol. 39, no. 10-11, pp. 2981-2993, 2015.

[32] J.-H. Kang and Y.-D. Kim, "Coordination of inventory and transportation managements in a two-level supply chain," International Journal of Production Economics, vol. 123, no. 1, pp. 137-145, 2010.

[33] H.-C. Hwang, "Inventory replenishment and inbound shipment scheduling under a minimum replenishment policy," Transportation Science, vol. 43, no. 2, pp. 244-264, 2009.

[34] H.-C. Hwang, "Economic lot-sizing for integrated production and transportation," Operations Research, vol. 58, no. 2, pp. 428444, 2010.

[35] S.-D. Lee and Y.-C. Fu, "Joint production and shipment lot sizing for a delivery price-based production facility," International Journal of Production Research, vol. 51, no. 20, pp. 6152-6162, 2013.

[36] S. Makkar and P. C. Jha, "Procurement-distribution model for perishable items with quantity discounts incorporating freight policies under fuzzy environment," Yugoslav Journal of Operations Research, vol. 23, no. 2, pp. 183-196, 2013.

[37] G. Y. Ke, J. H. Bookbinder, and D. M. Kilgour, "Coordination of transportation and quantity discount decisions, with coalition 
formation," International Journal of Production Research, vol. 52, no. 17, pp. 5115-5130, 2014.

[38] W. A. Jauhari and K. Winingsih, "Cooperative inventory model for vendor-buyer system with quantity and freight discounts," International Journal of Services and Operations Management, vol. 25, no. 4, pp. 508-530, 2016.

[39] Ş. İ. Birbil, K. Bülbül, J. B. Frenk, and H. M. Mulder, "On EOQ cost models with arbitrary purchase and transportation costs," Journal of Industrial and Management Optimization, vol. 11, no. 4, pp. 1211-1245, 2015. 


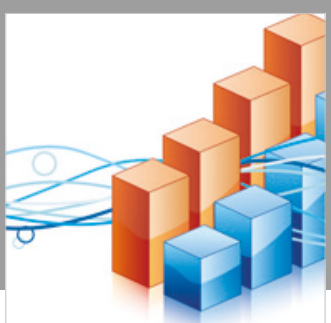

Advances in

Operations Research

\section{-n-m}
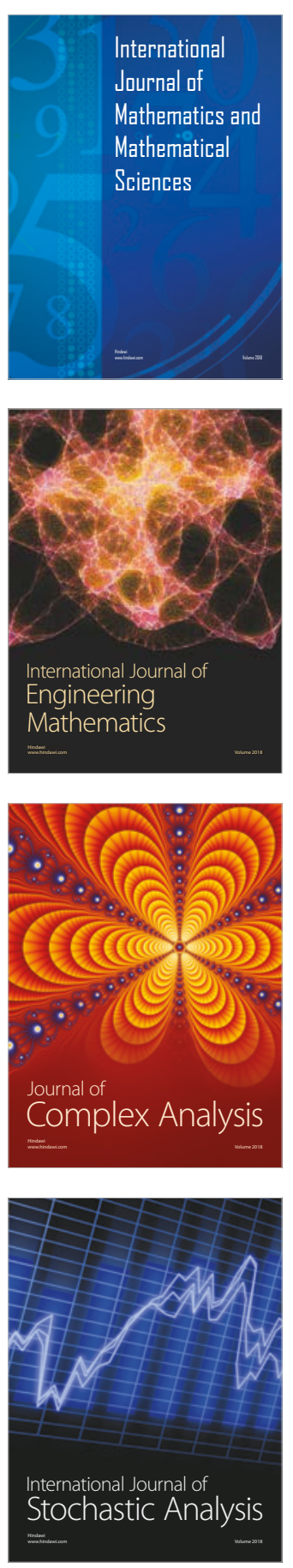
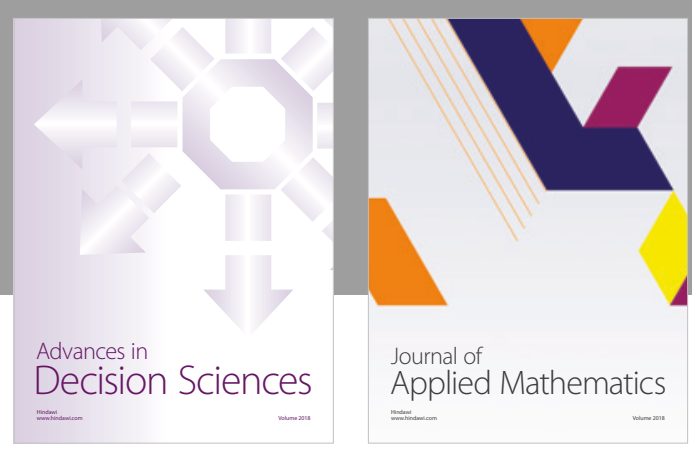

Journal of

Applied Mathematics
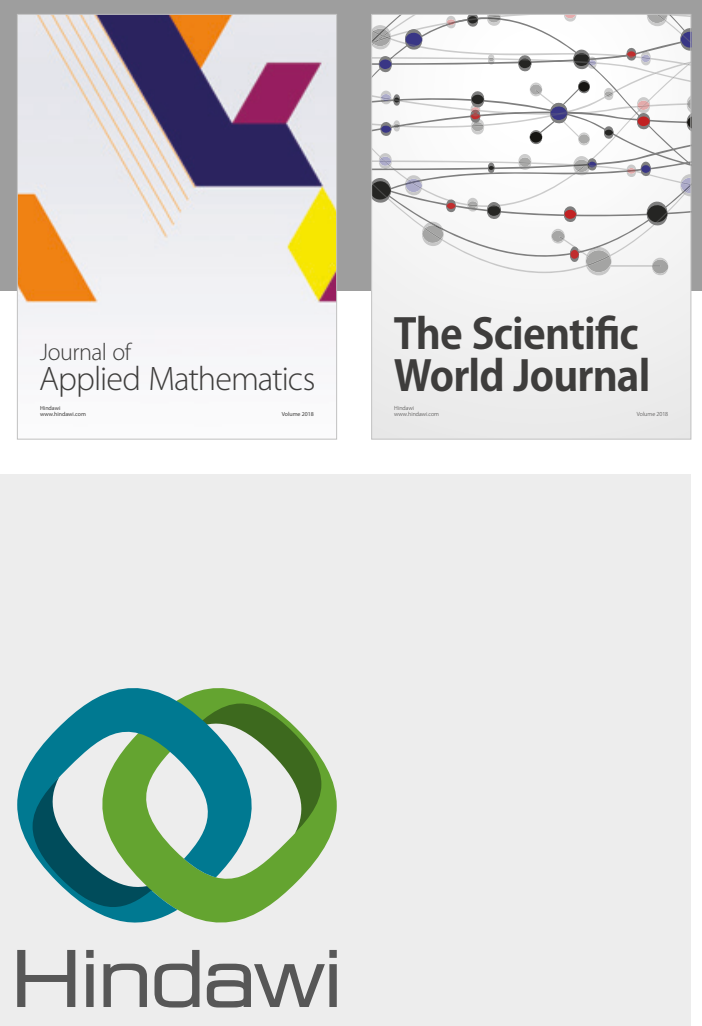

Submit your manuscripts at

www.hindawi.com

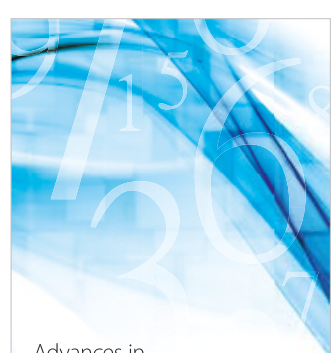

Advances in
Numerical Analysis
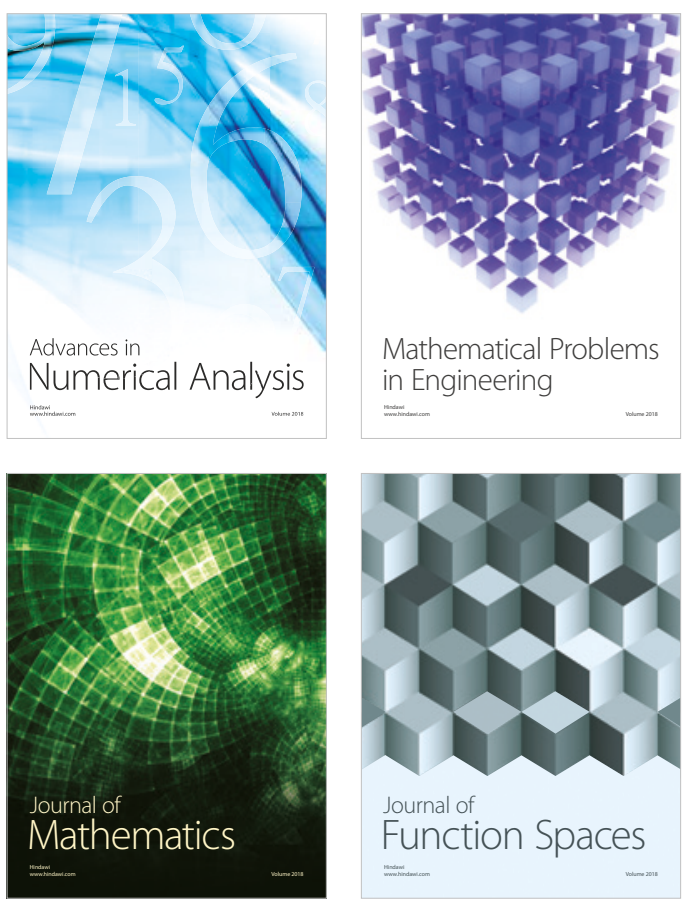

Mathematical Problems in Engineering

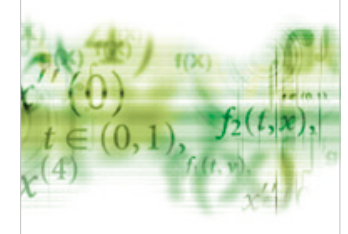

International Journal of

Differential Equations

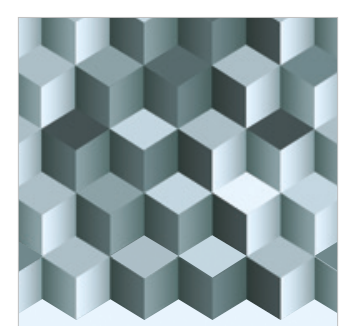

Journal of

Function Spaces

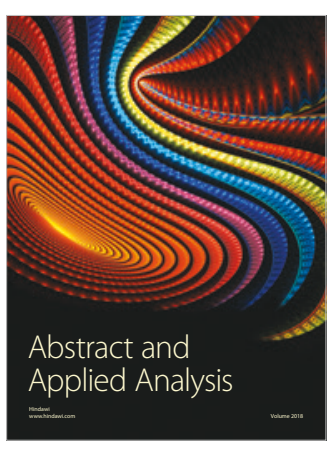

The Scientific

World Journal

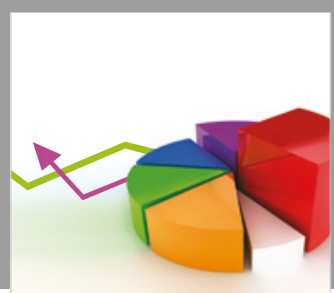

Journal of

Probability and Statistics
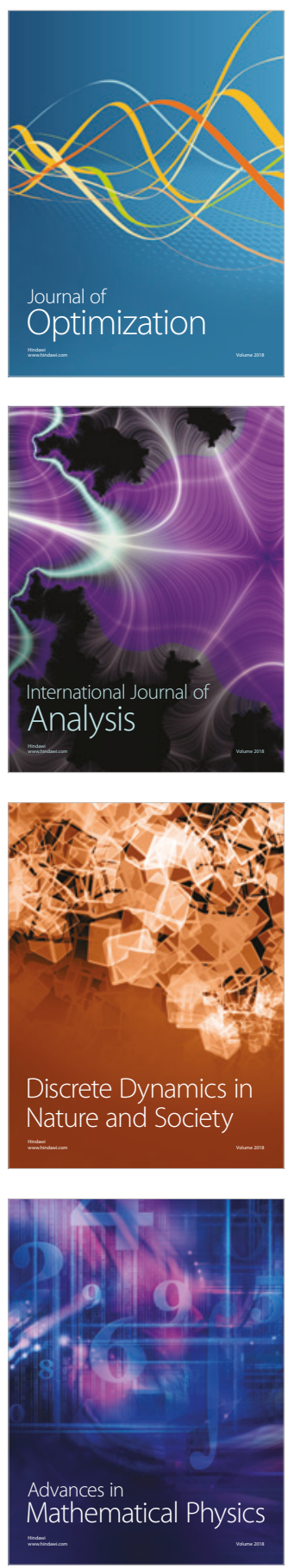